\title{
The super complex Frobenius theorem
}

\author{
by C. Denson Hill and Santiago R. Simanca (Stony Brook, N.Y.)
}

Abstract. We formulate and prove a super analogue of the complex Frobenius theorem of Nirenberg.

1. Introduction. We shall discuss the super analogue of the complex Frobenius theorem of L. Nirenberg [9]. Let us first recall a special case of this classical result: suppose $X$ is a smooth real manifold of dimension $N$ without boundary, and locally there are prescribed smooth complex vector fields $P_{1}, \ldots, P_{n}$ which satisfy the conditions:

$$
\begin{gathered}
P_{1}, \ldots, P_{n}, \bar{P}_{1}, \ldots, \bar{P}_{n} \text { are linearly independent, } \\
{\left[P_{j}, P_{k}\right]=\sum_{i=1}^{n} \alpha_{j k}^{i} P_{i}} \\
{\left[P_{j}, \bar{P}_{k}\right]=\sum_{i=1}^{n} \beta_{j k}^{i} P_{i}+\sum_{i=1}^{n} \gamma_{j k}^{i} \bar{P}_{i}}
\end{gathered}
$$

for some smooth functions $\alpha_{j k}^{i}, \beta_{j k}^{i}$ and $\gamma_{j k}^{i}$. Here $2 n+d=N$ with $d \geq 0$. Then there exists a system of local real coordinates $\left(x^{1}, \ldots, x^{n}, y^{1}, \ldots, y^{n}\right.$, $\left.r^{1}, \ldots, r^{d}\right)$ such that, setting $z^{k}=x^{k}+\sqrt{-1} y^{k}$, and possibly replacing $\left\{P_{k}\right\}$ by a suitable nonsingular linear combination of the $P_{j}$ 's with smooth coefficients, one has

$$
P_{k}=\partial / \partial z^{k},
$$

with $r^{1}, \ldots, r^{d}$ playing the role of parameters. This means that $X$ is foliated by a real $d$-parameter family of complex manifolds of complex dimension $n$. Another way to state the hypothesis would be to say that we have an abstract Levi flat CR structure of type $\mathbb{C}^{n} \times \mathbb{R}^{d}$ on $X$. Our first task will be

1991 Mathematics Subject Classification: Primary 58A50.

Key words and phrases: graded-commutative algebras, supermanifolds, Levi flat super CR structure, locally direct sheaf, super real integrable distribution, super complex Frobenius structure, nilpotent element, derivations. 
to formulate, in Theorem 1, the super analogue of this special case. Then we shall discuss, in Theorem 2, the super analogue of the more general result of Nirenberg, which involves dropping the first hypothesis in (1.1) and allowing some of the $\left\{P_{k}\right\}$ to be real vector fields. For completeness we include a self-contained proof of the super version of the real Frobenius theorem. We also include a Corollary and a Lemma which might be of some independent interest.

Let $(X, \mathcal{A}, \alpha)$ be a real $C^{\infty}$ supermanifold of dimension $(N, M)$. Recall what this means [1], [4], [6], [7]: $X$ is a $C^{\infty}$ manifold of real dimension $N, \mathcal{A}$ is a sheaf over $X$ of $\mathbb{Z}_{2}$ graded-commutative algebras over $\mathbb{R}$, and the augmentation map $\alpha: \mathcal{A} \rightarrow C^{\infty}$ is a sheaf homomorphism of algebras. The following axiom must be satisfied, which gives a local splitting: there exists a basis $\{V\}$ for the open sets of $X$ such that for every $V$ there is an isomorphism $\beta_{V}$ which makes the diagram

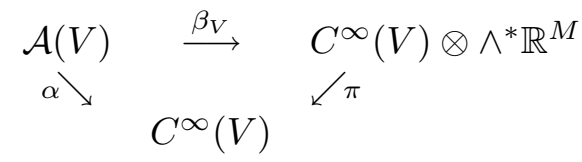

commutative. Here and in what follows we use the notation $\mathcal{S}(U)$ for the space of continuous sections over $U$ of a sheaf $\mathcal{S}$ over $X$. In the diagram above, $\pi$ is the natural projection.

A $\mathbb{Z}_{2}$ grading of $\mathcal{A}$ means that two subspaces $\mathcal{A}_{0}$ and $\mathcal{A}_{1}$ are fixed, the even and odd part respectively, such that $\mathcal{A}=\mathcal{A}_{0} \oplus \mathcal{A}_{1}$. The elements of $\mathcal{A}_{0}$ commute with all elements in $\mathcal{A}$ while the elements in $\mathcal{A}_{1}$ anticommute with all elements in $\mathcal{A}_{1}$. Let $\mathcal{N}$ be the subsheaf of nilpotent elements of $\mathcal{A}$. It follows from the above diagram that for any open set $U$ in $X$ there is a map tilde induced by $\alpha$ such that

$$
\mathcal{A}(U) \longrightarrow C^{\infty}(U) \stackrel{\cong}{\longrightarrow} / \mathcal{N}(U), \quad f \longrightarrow \widetilde{f} .
$$

Sections $x_{1}, \ldots, x_{N} \in \mathcal{A}_{0}(U)$ are called even coordinates if the functions $\widetilde{x}_{1}, \ldots, \widetilde{x}_{N} \in C^{\infty}(U)$ form a coordinate system in $U$ in the usual sense. Sections $\xi_{1}, \ldots, \xi_{k}$ of $\mathcal{A}_{1}(U)$ are algebraically independent if the product $\xi_{1} \ldots \xi_{k} \neq 0$. The odd dimension $M$ is defined as the smallest integer $j$ such that $\mathcal{N}^{j+1}=0$. Then $M$ algebraically independent sections $\xi_{1}, \ldots, \xi_{M} \in$ $\mathcal{A}_{1}(U)$ are said to form odd coordinates. Therefore a section $f$ of $\mathcal{A}(U)$, called a $C^{\infty}$ superfunction, can be written as $f=\sum_{\mu} f_{\mu}(x) \xi^{\mu}$ where $f_{\mu}(x) \in$ $\mathcal{A} / \mathcal{N}(U)$. Here $\mu=\left(\mu_{1}, \ldots, \mu_{M}\right)$ is a multi-index with $\mu_{j}=0$ or 1 , and $\xi^{\mu}=\xi_{1}^{\mu_{1}} \ldots \xi_{M}^{\mu_{M}}$. By $(1.2)$, in a sufficiently small open set $U$, we can think of a superfunction as an expression of the form

$$
f=\sum_{\mu} f_{\mu} \xi^{\mu}
$$


with $f_{\mu} \in C^{\infty}(U)$. The grading is thus determined by $f \in \mathcal{A}(U)_{0} \Leftrightarrow f=$ $\sum_{\mu:|\mu| \text { even }} f_{\mu} \xi^{\mu}$ while $f \in \mathcal{A}(U)_{1} \Leftrightarrow f=\sum_{\mu:|\mu| \text { odd }} f_{\mu} \xi^{\mu}$. The collection $\left\{x_{i}, \xi_{j}\right\}$ will be called a supercoordinate system.

The algebra of derivations Der $\mathcal{A}$ has a natural $\mathbb{Z}_{2}$ grading. Namely a derivation $D$ has degree $j$ iff $D(f g)=(D f) g+(-1)^{j \operatorname{deg} f} f(D g)$ for all homogeneous $f, g \in \mathcal{A}$. Given $D \in(\operatorname{Der} \mathcal{A}(U))_{0}$ the nilpotent set $\mathcal{N}$ is stable under $D$, and therefore it generates a derivation $\widetilde{D}$ of the quotient $\mathcal{A} / \mathcal{N}(U)$. Thus $\widetilde{D}$ can be thought of as a vector field over $U$. The correspondence

$$
(\operatorname{Der} \mathcal{A}(U))_{0} \rightarrow \operatorname{Der} C^{\infty}(U), \quad D \rightarrow \widetilde{D},
$$

is a Lie algebra epimorphism and we have $\widetilde{D F}=\widetilde{D} \widetilde{f}$ for all $f \in \mathcal{A}(U)$. In a supercoordinate system $\left\{x_{i}, \xi_{j}\right\}$ we have the partial derivations $\partial / \partial x_{i}$ and $\partial / \partial \xi_{j}$ defined by

$$
\frac{\partial}{\partial x_{i}}\left(f_{\mu} \xi^{\mu}\right)=\frac{\partial f_{\mu}}{\partial x_{i}} \xi^{\mu}, \quad \frac{\partial}{\partial \xi_{j}}\left(f_{\mu} \xi^{\mu}\right)=\mu_{j}(-1)^{p} f_{\mu} \xi^{\mu^{\prime}},
$$

where $p=\mu_{1}+\mu_{2}+\ldots+\mu_{j-1}$ and $\xi^{\mu^{\prime}}=\xi_{1}^{\mu_{1}} \cdot \xi_{2}^{\mu_{2}} \ldots \xi_{j}^{\mu_{j}-1} \ldots \xi_{M}^{\mu_{M}}$. The even $\left\{\partial / \partial x_{i}\right\}$ commute and can be thought of as classical partial derivatives, whereas the odd $\left\{\partial / \partial \xi_{j}\right\}$ anticommute. Locally $\operatorname{Der} \mathcal{A}$ is a free $\mathcal{A}(U)$-module with basis $\left\{\partial / \partial x_{i}, \partial / \partial \xi_{j}\right\}$, i.e., linear combinations of these basis elements with $C^{\infty}$ superfunction coefficients.

We use extension by real linearity to complexify both the superalgebra $\mathcal{A}$ and the algebra of derivations $\operatorname{Der} \mathcal{A}$; let $\mathcal{A}_{\mathbb{C}}$ and $\operatorname{Der}_{\mathbb{C}} \mathcal{A}_{\mathbb{C}}$ denote the respective complexifications. We also use real linearity to extend the supercommutator defined by

$$
[X, Y]=X Y-(-1)^{d(X) d(Y)} Y X
$$

for homogeneous derivations $X, Y$, where $d(X)$ denotes the degree of $X$.

We make the following definition: suppose $2 n+l=N$ and $2 m+k=M$, with $l, k \geq 0$. Then a Levi flat super $C R$ structure on $(X, \mathcal{A}, \alpha)$ of $\mathrm{CR}$ dimension $(n, m)$ (or $\mathrm{CR}$ codimension $(l, k))$ consists in the prescription of a locally direct subsheaf $\mathcal{H}$ of the sheaf $\operatorname{Der}_{\mathbb{C}} \mathcal{A}_{\mathbb{C}}$ of $\mathcal{A}_{\mathbb{C}}$-modules over $X$ of rank $(n, m)$. This means that locally the sections of $\mathcal{H}$ form a direct summand in the free module of local sections $\operatorname{Der}_{\mathbb{C}} \mathcal{A}_{\mathbf{C}}$, which is generated by $n$ even and $m$ odd linearly independent derivations. We also require that

$$
\mathcal{H} \cap \overline{\mathcal{H}}=0, \quad[\mathcal{H}, \mathcal{H}] \subset \mathcal{H}, \quad[\mathcal{H}, \overline{\mathcal{H}}] \subset \mathcal{H}+\overline{\mathcal{H}} .
$$

Thus locally in $U$, the prescription of $\mathcal{H}$ is equivalent to prescribing a basis for sections of $\mathcal{H}$ of the form $\left\{P_{1}, \ldots, P_{n+m}\right\}=\left\{L_{1}, \ldots, L_{n}, M_{1}, \ldots, M_{m}\right\}$, where $L_{1}, \ldots, L_{n}$ are of degree 0 and $M_{1}, \ldots, M_{m}$ are of degree 1 . In terms of the basis $\left\{P_{i}\right\}$, the conditions above are written as in (1.1), except one must use the supercommutator, $n$ is replaced by $n+m$, and the coefficients $\alpha_{j k}^{i}$, 
$\beta_{j k}^{i}, \gamma_{j k}^{i}$ are $C^{\infty}$ superfunctions. In terms of the $\left\{L_{i}, M_{j}\right\}$ these integrability conditions become:

$$
\begin{gathered}
L_{1}, \ldots, L_{n}, \bar{L}_{1}, \ldots, \bar{L}_{n}, M_{1}, \ldots, M_{m}, \bar{M}_{1}, \ldots, \bar{M}_{m} \\
\text { are linearly independent, } \\
{\left[L_{i}, L_{j}\right]=a_{i j}^{r} L_{r}+b_{i j}^{s} M_{s},} \\
{\left[M_{p}, M_{q}\right]=c_{p q}^{r} L_{r}+d_{p q}^{s} M_{s},} \\
{\left[L_{i}, M_{q}\right]=e_{i q}^{r} L_{r}+f_{i q}^{s} M_{s},} \\
{\left[L_{i}, \bar{L}_{j}\right]=l_{i j}^{r} L_{r}-\bar{l}_{j i}^{r} \bar{L}_{r}+m_{i j}^{s} M_{s}-\bar{m}_{j i}^{s} \bar{M}_{s},} \\
{\left[M_{p}, \bar{M}_{q}\right]=n_{p q}^{r} L_{r}+\bar{n}_{q p}^{r} \bar{L}_{r}+o_{p q}^{s} M_{s}+\bar{o}_{q p}^{s} \bar{M}_{s},} \\
{\left[L_{i}, \bar{M}_{q}\right]=p_{i q}^{j} L_{j}+r_{i q}^{j} \bar{L}_{j}+s_{i q}^{l} M_{l}+t_{i q}^{l} \bar{M}_{l},}
\end{gathered}
$$

where the coefficients in these expressions are sections of $\mathcal{A}_{\mathbb{C}}$. Note that the middle equations in (1.5) and (1.6) involve anticommutators. Also the last equation in (1.6) implies that

$$
\left[\bar{L}_{i}, M_{q}\right]=\bar{r}_{i q}^{j} L_{j}+\bar{p}_{i q}^{j} \bar{L}_{j}+\bar{t}_{i q}^{l} M_{l}+\bar{s}_{i q}^{l} \bar{M}_{l} .
$$

When $l, k=0$ the conditions (1.6) are automatically true, since $\operatorname{Der}_{\mathbb{C}} \mathcal{A}_{\mathbb{C}}$ has rank $N+M=2(n+m)$; in that case we have an integrable super almost complex structure on $(X, \mathcal{A}, \alpha)$. This case has been discussed by A. McHugh [8] and the authors [3]. Now we can state our first result.

TheOREM 1. Let $\mathcal{H}$ be a Levi flat super $C R$ structure on $(X, \mathcal{A}, \alpha)$ of $C R$ dimension $(n, m)$. Then given a point $p \in X$, there is a neighborhood $U$ of $p$ in $X$, and a supercoordinate system on $U$ with even coordinates $\left(x^{1}, \ldots, x^{n}, y^{1}, \ldots, y^{n}, r^{1}, \ldots, r^{l}\right)$ and odd coordinates $\left(\xi^{1}, \ldots, \xi^{m}, \eta^{1}, \ldots\right.$, $\left.\eta^{m}, \varrho^{1}, \ldots, \varrho^{k}\right)$, so that setting $z^{j}=x^{j}+\sqrt{-1} y^{j}, \zeta^{j}=\xi^{j}+\sqrt{-1} \eta^{j}$, there is a basis in $U$ for $\mathcal{H}$ of the form

$$
L_{i}=\partial / \partial z^{i}, \quad M_{q}=\partial / \partial \zeta^{q} .
$$

Here the $r^{i}, \varrho^{j}$ play the role of parameters, and the derivations $\partial / \partial z^{i}, \partial / \partial \zeta^{q}$ are defined by the usual formulas in terms of the real derivations:

$$
\frac{\partial}{\partial z^{i}}=\frac{1}{2}\left(\frac{\partial}{\partial x^{i}}-\sqrt{-1} \frac{\partial}{\partial y^{i}}\right), \quad \frac{\partial}{\partial \zeta^{q}}=\frac{1}{2}\left(\frac{\partial}{\partial \xi^{q}}-\sqrt{-1} \frac{\partial}{\partial \eta^{q}}\right) .
$$

In order to formulate the super analogue of the general result of Nirenberg we make the following definition: suppose $2 n+l+e=N$ and $2 m+$ $k+d=M$, with $l, k, e, d \geq 0$. Then by a super complex Frobenius structure of type $(n, e, m, d)$ on $(X, \mathcal{A}, \alpha)$ we shall mean that a locally direct subsheaf 
$\mathcal{D}$ of $\operatorname{Der}_{\mathbb{C}} \mathcal{A}_{\mathbb{C}}$ has been prescribed which satisfies

$$
\begin{gathered}
\operatorname{rank} \mathcal{D}=(n+e, m+d), \quad \operatorname{rank} \mathcal{D} \cap \overline{\mathcal{D}}=(e, d), \\
{[\mathcal{D}, \mathcal{D}] \subset \mathcal{D}, \quad[\mathcal{D}, \overline{\mathcal{D}}] \subset \mathcal{D}+\overline{\mathcal{D}}}
\end{gathered}
$$

This means that:

1. Locally there is a basis for sections of $\mathcal{D}$ of the form $\left\{L_{1}, \ldots, L_{n}\right.$, $\left.X_{1}, \ldots, X_{e}, M_{1}, \ldots, M_{m}, Y_{1}, \ldots, Y_{d}\right\}$ where the $L_{i}, X_{j}$ are even, the $M_{i}$, $Y_{j}$ are odd, and the $X_{i}, Y_{j}$ are real.

2. The collection $\left\{L_{1}, \ldots, L_{n}, \bar{L}_{1}, \ldots, \bar{L}_{n}, X_{1}, \ldots, X_{e}, M_{1}, \ldots, M_{m}, \bar{M}_{1}\right.$, $\left.\ldots, \bar{M}_{m}, Y_{1}, \ldots, Y_{d}\right\}$ is linearly independent.

3. The supercommutator of any two derivations from the list in 1 is a linear combination of the list from 1 ; and the supercommutator of any two derivations from the list in 2 is a linear combination of the list from 2 .

Notice that when $d=e=0$, we have a Levi flat super CR structure of CR dimension $(n, m)$.

TheOREM 2. Let $\mathcal{D}$ be a complex Frobenius structure on $(X, \mathcal{A}, \alpha)$ of type $(n, e, m, d)$. Then given a point $p \in X$, there is a neighborhood $U$ of $p$ in $X$, and a supercoordinate system on $U$ with even coordinates $\left(x^{1}, \ldots, x^{n}, y^{1}\right.$, $\left.\ldots, y^{n}, r^{1}, \ldots, r^{l}, s^{1}, \ldots, s^{e}\right)$ and odd coordinates $\left(\xi^{1}, \ldots, \xi^{m}, \eta^{1}, \ldots, \eta^{m}\right.$, $\left.\varrho^{1}, \ldots, \varrho^{k}, \sigma^{1}, \ldots, \sigma^{d}\right)$, so that setting $z^{j}=x^{j}+\sqrt{-1} y^{j}, \zeta^{j}=\xi^{j}+\sqrt{-1} \eta^{j}$, there is a basis in $U$ for $\mathcal{D}$ of the form

$$
L_{i}=\frac{\partial}{\partial z^{i}}, \quad M_{i}=\frac{\partial}{\partial \zeta^{i}}, \quad X_{i}=\frac{\partial}{\partial s^{i}}, \quad Y_{i}=\frac{\partial}{\partial \sigma^{i}} .
$$

Here the $r^{i}, \varrho^{j}$ play the role of parameters.

2. Related results. We will need to utilize some closely related results which we discuss in this section.

First we shall need the super analogue of the real Frobenius theorem, which we shall formulate and prove since we have not been able to find a proof in the literature. Suppose $n+l=N$ and $m+k=M$, with $l, k \geq 0$. Then a super real integrable distribution on $(X, \mathcal{A}, \alpha)$ of type $(n, m)$ consists in the prescription of a locally direct subsheaf $\mathcal{J}$ of the sheaf Der $\mathcal{A}$ of $\mathcal{A}$-modules over $X$ of rank $(n, m)$. We require the integrability condition

$$
[\mathcal{J}, \mathcal{J}] \subset \mathcal{J}
$$

where of course we use the supercommutator. Thus locally the sections of $\mathcal{J}$ form a direct summand in the free module of local sections Der $\mathcal{A}$, which is generated by $n$ even and $m$ odd real linearly independent derivations. So locally in $U$, the prescription of $\mathcal{J}$ is equivalent to prescribing a basis for 
the sections of $\mathcal{J}$ of the form $\left\{X_{1}, \ldots, X_{n}, Y_{1}, \ldots, Y_{m}\right\}$, where $X_{1}, \ldots, X_{n}$ are of degree 0 and $Y_{1}, \ldots, Y_{m}$ are of degree 1 . In terms of this basis the integrability condition is written as:

$$
\begin{aligned}
{\left[X_{i}, X_{j}\right] } & =a_{i j}^{r} X_{r}+b_{i j}^{s} Y_{s}, \\
{\left[Y_{p}, Y_{q}\right] } & =c_{p q}^{r} X_{r}+d_{p q}^{s} Y_{s}, \\
{\left[X_{i}, Y_{q}\right] } & =e_{i q}^{r} X_{r}+f_{i q}^{s} Y_{s},
\end{aligned}
$$

with coefficients that are real $C^{\infty}$ superfunctions. Since the $Y_{j}$ are odd, the anticommutator occurs in the middle expression above.

THEOREM 3. Let $\mathcal{J}$ be a super real integrable distribution on $(X, \mathcal{A}, \alpha)$ of type $(n, m)$. Then given a point $p \in X$ there is a neighborhood $U$ in $X$ and a supercoordinate system on $U$ with even coordinates $\left(x^{1}, \ldots, x^{n}, r^{1}, \ldots, r^{l}\right)$ and odd coordinates $\left(\xi^{1}, \ldots, \xi^{m}, \varrho^{1}, \ldots, \varrho^{k}\right)$ so that in $U$ the sections of $\mathcal{J}$ are spanned by the derivations

$$
\partial / \partial x^{i}, \quad 1 \leq i \leq n, \quad \partial / \partial \xi^{q}, \quad 1 \leq q \leq m .
$$

Proof. Using (1.3) we associate to the even derivations $X_{1}, \ldots, X_{n} \in$ Der $\mathcal{A}(U)$ the $C^{\infty}$ vector fields $\mathcal{X}_{j}=\tilde{X}_{j}$. Since the derivations $\left[X_{i}, X_{j}\right]$ are even and the $Y_{i}$ are odd, the sections $b_{i j}^{s}$ in (2.8) must be nilpotent. Thus the integrability condition implies that $\left\{\mathcal{X}_{1}, \ldots, \mathcal{X}_{n}\right\}$ span a classical integrable distribution $\widetilde{\mathcal{J}}$ of $n$ planes in $U$, i.e., $[\widetilde{\mathcal{J}}, \widetilde{\mathcal{J}}] \subset \widetilde{\mathcal{J}}$ where [ , ] is now the usual bracket of vector fields in $U$. Applying the classical Frobenius theorem we find coordinates $\left(\widetilde{x}^{1}, \ldots, \widetilde{x}^{n}, \widetilde{r}^{1}, \ldots, \widetilde{r}^{l}\right)$ in a possibly smaller neighborhood $U$, so that $\widetilde{\mathcal{J}}$ is spanned by $\partial / \partial \widetilde{x}^{j}, 1 \leq j \leq n$. Using the augmentation map, we lift to some choice of even coordinates $\left(x^{1}, \ldots, x^{n}, r^{1}, \ldots, r^{l}\right)$ in the supermanifold. After replacing the original $X_{i}$ by suitable linear combinations which we rename $X_{i}$, we have

$$
X_{i}=\partial / \partial x^{i}+A,
$$

for some $A$ in the kernel of (1.3) on $U$. On the other hand, we can select odd coordinates $\xi^{1}, \ldots, \xi^{m+k}$ such that, after replacing the $Y_{i}$ by some suitable linear combinations, which we rename $Y_{i}$, we have

$$
Y_{i}=\frac{\partial}{\partial \xi^{i}}+\sum_{j=1}^{k} f_{i j} \frac{\partial}{\partial \xi^{j+m}}+B, \quad j=1, \ldots, m,
$$

for some real even sections $f_{i j}$, where the derivation $B$ is in the span of $\mathcal{N} \operatorname{Der} \mathcal{A}(U)$. If we perform the linear change $\xi^{j} \rightarrow \xi^{j}, 1 \leq j \leq m, \xi^{s+m} \rightarrow$ $f_{j s} \xi^{j}+\varrho^{s}, 1 \leq s \leq k$, we see that $f_{j k}$ can be assumed to be zero. Thus we have supercoordinates $(x, r, \xi, \varrho)$ such that, for some nilpotent sections $A_{j}^{i, r}$, 
$B_{j}^{i, r}, C_{j}^{q, r}, D_{j}^{q, r}$, the derivations

$$
\begin{aligned}
& X_{i}=\frac{\partial}{\partial x^{i}}+A_{i}^{j, 0} \frac{\partial}{\partial x^{j}}+B_{i}^{s, 0} \frac{\partial}{\partial r^{s}}+C_{i}^{j, 0} \frac{\partial}{\partial \xi^{j}}+D_{i}^{q, 0} \frac{\partial}{\partial \varrho^{q}}, \\
& Y_{j}=\frac{\partial}{\partial \xi^{j}}+A_{j}^{i, 1} \frac{\partial}{\partial x^{i}}+B_{j}^{i, 1} \frac{\partial}{\partial r^{i}}+C_{j}^{s, 1} \frac{\partial}{\partial \xi^{s}}+D_{j}^{q, 1} \frac{\partial}{\partial \varrho^{q}}
\end{aligned}
$$

span the distribution $\mathcal{J}$. Suppose that all of the coefficients labeled $A$ and $C$ above are in $\mathcal{N}^{p}$. The sections $A_{i}^{j, 0}$ and $C_{j}^{q, 1}$ are even, while the sections $A_{j}^{i, 1}$ and $C_{i}^{q, 0}$ are odd. So by changing our derivations to

$$
X_{i}-\left(A_{i}^{j, 0} X_{j}+C_{i}^{q, 0} Y_{q}\right), \quad Y_{j}-\left(A_{j}^{i, 1} X_{j}+C_{j}^{q, 1} Y_{q}\right),
$$

we obtain a new family, which we rename $\left\{X_{i}, Y_{j}\right\}$, generating the same structure $\mathcal{J}$ and for which (2.9) holds with all of the coefficients labeled $A$ and $C$ in $\mathcal{N}^{p+1}$. Iterating the procedure $M+1=m+k+1$ times we obtain derivations of the form

$$
\begin{aligned}
X_{i} & =\frac{\partial}{\partial x^{i}}+B_{i}^{s, 0} \frac{\partial}{\partial r^{s}}+D_{i}^{q, 0} \frac{\partial}{\partial \varrho^{q}}, \\
Y_{j} & =\frac{\partial}{\partial \xi^{j}}+B_{j}^{i, 1} \frac{\partial}{\partial r^{i}}+D_{j}^{q, 1} \frac{\partial}{\partial \varrho^{q}},
\end{aligned}
$$

which $\operatorname{span} \mathcal{J}$.

To eliminate the remaining coefficients in the expression above we proceed also by induction on their order of nilpotency. Assume (2.10) holds with coefficients in $\mathcal{N}^{l}$ modulo $\mathcal{N}^{l+1}$. We have proven the result for $l=1$. Assuming it holds for any $k \leq l$ we want to show it is true for $l+1$. Suppose $l$ is even, as the cases where $l$ is even or odd differ slightly.

We expand $X_{i}, Y_{j}$ modulo nilpotent terms of degree $l+1$ times derivations. Looking at the $\mathbb{Z}_{2}$ grading, we conclude that the coefficients $D_{i}^{q, 0}$ and $B_{j}^{i, 1}$ must be zero. Thus

$$
\begin{aligned}
X_{i} & =\frac{\partial}{\partial x^{i}}+B_{i}^{s, 0} \frac{\partial}{\partial r^{s}}, \\
Y_{j} & =\frac{\partial}{\partial \xi^{j}}+D_{j}^{q, 1} \frac{\partial}{\partial \varrho^{q}},
\end{aligned}
$$

The integrability condition applied to the bracket of these derivations implies readily that

$$
\frac{\partial B_{j}^{s, 0}}{\partial x^{i}}=\frac{\partial B_{i}^{s, 0}}{\partial x^{j}}, \quad \frac{\partial B_{j}^{s, 0}}{\partial \xi^{i}}=0, \quad \frac{\partial D_{k}^{q, 1}}{\partial \xi^{j}}=-\frac{\partial D_{j}^{q, 1}}{\partial \xi^{k}} .
$$

Thus we can solve the equation

$$
\nabla_{x} v^{s}=\left(B_{1}^{s, 0}, \ldots, B_{n}^{s, 0}\right),
$$


with a solution independent of $\xi$. We then switch coordinates by $x \rightarrow x$, $r^{s} \rightarrow v^{s}+r^{s}, \xi \rightarrow \xi, \varrho \rightarrow \varrho$. Then our spanning set can be expressed as in (2.10), and it is such that (2.11) holds with the coefficient $B_{i}^{s, 0}$ zero. Now suppose that $D_{j}^{q, 1}$ is homogeneous in $\xi$. The condition imposed by the integrability implies that $-\xi^{j} \partial_{\xi^{k}} D_{j}^{q, 1}=\xi^{j} \partial_{\xi^{j}} D_{k}^{q, 1}=\lambda D_{k}^{q, 1}$, where $\lambda$ is the degree of homogeneity in $\xi$. Thus, if we now change our supercoordinates by $x \rightarrow x, r \rightarrow r, \xi \rightarrow \xi, \varrho^{q} \rightarrow(\lambda+1)^{-1} D_{j}^{q, 1} \xi^{j}+\varrho^{q}$, the conclusion about the $X_{i}$ remains unchanged, and in the new coordinate system, (2.11) holds with the coefficients $D_{j}^{q, 1}$ equal to zero. In general $D_{j}^{q, 1}$ is the sum of homogeneous terms, and we can make them vanish by a similar change, giving the appropriate weight corresponding to the homogeneity of each one of them. This completes the proof of Theorem 3.

Remark 1. If one has basis elements $\left\{X_{1}, \ldots, X_{n}, Y_{1}, \ldots, Y_{m}\right\}$ for $\mathcal{J}$ such that the brackets in (2.8) are all zero, one can find a supercoordinate system $(x, r, \xi, \varrho)$ in which the basis elements are the coordinate derivations $\partial / \partial x^{i}, \partial / \partial \xi^{q}$. One can prove this statement by an argument which follows almost verbatim the one above, where one needs to use the super version of the Poincaré lemma with parameters. This is true since the proof of the super Poincaré lemma in [4] goes through with parameters. Furthermore, the super real Frobenius theorem discussed above also holds with smooth dependence on parameters.

Before going any further, we discuss a corollary of Theorem 3 which is analogous to the classical construction of an integral manifold of a family of linearly independent vector fields $\left\{X_{1}, \ldots, X_{k}\right\}$, on an open set $U$ in $\mathbb{R}^{n}$, which commute. Indeed, if we have a coordinate system $(t, x)$ such that

$$
X_{i}=\frac{\partial}{\partial t^{i}}+a_{i}^{s} \frac{\partial}{\partial x^{s}},
$$

then one can solve the initial value problem

$$
\left\{\begin{array}{l}
\frac{\partial x^{s}}{\partial t^{i}}=a_{i}^{s}(t, x), \quad 1 \leq i \leq k, \quad 1 \leq s \leq n-k, \\
x(0)=x_{0} .
\end{array}\right.
$$

The integrability conditions for this system are exactly the conditions on the coefficients $a_{i}^{s}$ which arise from the fact that $\left[X_{i}, X_{j}\right]=0$. The integral manifold through the point $\left(0, x_{0}\right)$ is defined by $F_{X_{1}}^{t^{1}} \circ \ldots \circ F_{X_{k}}^{t^{k}}\left(0, x_{0}\right)=$ $\left(t^{1}, \ldots, t^{k}, x^{1}(t), \ldots, x^{n-k}(t)\right)$, where $F_{Y}^{t}$ is the flow of the vector field $Y$ at time $t$.

We discuss the notion of "flow" of particular types of superderivations. Let $X$ be a basis for a real super distribution of rank $(1,0)$ which is integrable. According to the Theorem above, there exists a supercoordinate system $(x, \xi)$ where $X=\partial / \partial x^{1}$. We define its flow for even time $t$ in the 
coordinate system $(x, \xi)$ by

$$
F_{X}^{t}(x, \xi)=\left(x^{1}+t, x^{\prime}, \xi\right),
$$

where $x^{\prime}=\left(x^{2}, \ldots, x^{N}\right)$. On the other hand, if $Y$ is a basis for a real superdistribution of rank $(0,1)$ which is integrable, then there exists a supercoordinate system $(y, \eta)$ such that $Y=\partial / \partial \eta^{1}$. We define its flow for odd time $\tau$ in the coordinate system $(y, \eta)$ by

$$
F_{Y}^{\tau}(y, \eta)=\left(y, \eta^{1}+\tau, \eta^{\prime}\right),
$$

where $\eta^{\prime}=\left(\eta^{2}, \ldots, \eta^{M}\right)$.

Remark 2. In the terminology of Manin [7], the derivations $X$ and $Y$ above are rectifiable of type $(1,0)$ and $(0,1)$, respectively. There is yet a third type of derivation, rectifiable of type $(1,1)$, for which a purely even or purely odd flow will not work. The typical model in supercoordinates $(x, \xi)$ of such a derivation is $\partial_{\xi^{1}}+\xi^{1} \partial_{x^{1}}$. In this case we refer the reader to [5].

One then can define the notion of a super Lie derivative as follows: for the derivation $X$ above, we set

$$
\mathcal{L}_{X} f(x, \xi)=\lim _{t \rightarrow 0} \frac{f\left(F_{X}^{t}(x, \xi)\right)-f(x, \xi)}{t},
$$

where the limit is computed in a natural topology on superfunctions which one can introduce having chosen the supercoordinates $(x, \xi)$. It is then elementary to check that

$$
\mathcal{L}_{X} f(x, \xi)=(X f)(x, \xi) .
$$

For the odd derivation $Y$ the situation is only slightly more complicated because division by the odd parameter $\tau$ is not defined. However, we can use the evaluation map $e_{\tau}$ (sometimes called Berezin integral [2] in $\tau$, a term which we refrain from using as various authors mean different things by such). This map acts as a superderivation. Indeed, if we separate the $\tau$ dependence in $f$ by writing $f=f_{0}+\tau f_{1}$, then

$$
e_{\tau}(f)=f_{1} \text {. }
$$

We therefore define

$$
\mathcal{L}_{Y} f(y, \eta)=e_{\tau}\left(f\left(F_{Y}^{\tau}(y, \eta)\right),\right.
$$

and once again we get

$$
\mathcal{L}_{Y} f(y, \eta)=(Y f)(y, \eta) .
$$

With these definitions set forth for rectifiable derivations of type $(1,0)$ and $(0,1)$, the classical result that the flows commute if and only if the vector fields commute holds with the appropriate modifications. One must use flows of real time for the first type of derivations and flows of odd time 
for the second, while the notion of commutativity is computed using the superbrackets of derivations.

Our corollary then goes as follows: suppose $\left\{X_{1}, \ldots, X_{n}, Y_{1}, \ldots, Y_{m}\right\}$ is a basis for a real superdistribution of rank $(n, m)$ which in a supercoordinate system $(t, x, \tau, \xi)$ can be expressed as

$$
\begin{aligned}
& X_{k}=\frac{\partial}{\partial t^{k}}+a_{k}^{0 s} \frac{\partial}{\partial x^{s}}+b_{k}^{0 j} \frac{\partial}{\partial \xi^{j}}, \\
& Y_{k}=\frac{\partial}{\partial \tau^{k}}+a_{k}^{1 s} \frac{\partial}{\partial x^{s}}+b_{k}^{1 j} \frac{\partial}{\partial \xi^{j}} .
\end{aligned}
$$

We assume further that

$$
\left[X_{i}, X_{j}\right]=0, \quad\left[X_{i}, Y_{l}\right]=0, \quad\left[Y_{l}, Y_{j}\right]=0 .
$$

These conditions together suffice to conclude that each even derivation $X_{i}$ is rectifiable of type $(1,0)$ while each odd derivation $Y_{j}$ is rectifiable of type $(0,1)$. We thus have flows of even and odd time, respectively.

Corollary 1. Let $X_{k}, Y_{l}$ be superderivations which locally in $U$ can be expressed as in (2.12) and satisfy (2.13). Then the initial value problem

$$
\begin{aligned}
\frac{\partial x^{s}}{\partial t^{k}} & =a_{k}^{0 s}(t, x, \tau, \xi), & \frac{\partial \xi^{s}}{\partial t^{k}} & =b_{k}^{0 s}(t, x, \tau, \xi), \\
\frac{\partial x^{s}}{\partial \tau^{k}} & =a_{k}^{1 s}(t, x, \tau, \xi), & \frac{\partial \xi^{s}}{\partial \tau^{k}} & =b_{k}^{1 s}(t, x, \tau, \xi), \\
x(0,0) & =x_{0}, & \xi(0,0) & =\xi_{0},
\end{aligned}
$$

has a smooth unique local solution $x\left(t, x_{0}, \tau, \xi_{0}\right), \xi\left(t, x_{0}, \tau, \xi_{0}\right)$.

Proof. Each of the conditions $\left[X_{k}, X_{r}\right]=0,\left[X_{k}, Y_{l}\right]=0$ and $\left[Y_{l}, Y_{j}\right]=0$ gives rise to two conditions; the six of them are:

$$
\begin{aligned}
& \partial_{t^{k}} a_{r}^{0 s}-\partial_{t^{r}} a_{k}^{0 s}+a_{k}^{0 l} \partial_{x^{l}} a_{r}^{0 s}-a_{r}^{0 l} \partial_{x^{l}} a_{k}^{0 s}+b_{k}^{0 j} \partial_{\xi^{j}} a_{r}^{0 s}-b_{r}^{0 j} \partial_{\xi^{j}} a_{k}^{0 s}=0, \\
& \partial_{t^{k}} b_{r}^{0 j}-\partial_{t^{r}} b_{k}^{0 j}+a_{k}^{0 l} \partial_{x^{l}} b_{r}^{0 j}-a_{r}^{0 l} \partial_{x^{l}} b_{k}^{0 j}+b_{k}^{0 l} \partial_{\xi^{l}} b_{r}^{0 j}-b_{r}^{0 l} \partial_{\xi^{l}} b_{k}^{0 j}=0, \\
& \partial_{t^{k}} a_{l}^{1 s}-\partial_{\tau^{l}} a_{k}^{0 s}+a_{k}^{0 j} \partial_{x^{j}} a_{l}^{1 s}-a_{l}^{1 j} \partial_{x^{j}} a_{k}^{0 s}+b_{k}^{0 j} \partial_{\xi^{j}} a_{l}^{1 s}-b_{l}^{1 j} \partial_{\xi^{j}} a_{k}^{0 s}=0, \\
& \partial_{t^{k}} b_{l}^{1 j}-\partial_{\tau^{l}} b_{k}^{0 j}+a_{k}^{0 s} \partial_{x^{s}} b_{l}^{1 j}-a_{l}^{1 s} \partial_{x^{s}} b_{k}^{0 j}+b_{k}^{0 s} \partial_{\xi^{s}} b_{l}^{1 j}-b_{l}^{1 s} \partial_{\xi^{s}} b_{k}^{0 j}=0, \\
& \partial_{\tau^{l}} a_{j}^{1 s}+\partial_{\tau^{j}} a_{l}^{1 s}+a_{l}^{1 k} \partial_{x^{k}} a_{j}^{1 s}+a_{j}^{1 k} \partial_{x^{k}} a_{l}^{1 s}+b_{j}^{1 k} \partial_{\xi^{k}} a_{l}^{1 s}+b_{l}^{1 k} \partial_{\xi^{k}} a_{j}^{1 s}=0, \\
& \partial_{\tau^{l}} b_{j}^{1 k}+\partial_{\tau^{j}} b_{l}^{1 k}+a_{l}^{1 s} \partial_{x^{s}} b_{j}^{1 k}+a_{j}^{1 s} \partial_{x^{s}} b_{l}^{1 k}+b_{l}^{1 s} \partial_{\xi^{s}} b_{j}^{1 k}+b_{j}^{1 s} \partial_{\xi^{s}} b_{l}^{1 k}=0 .
\end{aligned}
$$

These conditions correspond exactly with the integrability conditions of the system of equations stated in the corollary. With the flows defined above, the rest of the proof follows the classical argument.

Second we shall need the super analogue of the Newlander-Nirenberg theorem with parameters. We carefully formulate this notion for two reasons: one is that we need it to prove our results and the other is that it 
will shed light on the way the parameters come naturally into our work. By analogy the reader can formulate the parametric version of the super real Frobenius theorem alluded to in Remark 1.

Recall the notion of a super integrable almost complex structure on a real $C^{\infty}$ supermanifold $(Y, \mathcal{B}, \beta)$ of dimension $(2 n, 2 m)$. This is just the prescription of a locally direct subsheaf $\mathcal{F}$ of the sheaf $\operatorname{Der}_{\mathbb{C}} \mathcal{B}_{\mathbb{C}}$ of $\mathcal{B}_{\mathbb{C}^{-}}$ modules over $Y$, of rank $(n, m)$, which satisfies

$$
\mathcal{F} \cap \overline{\mathcal{F}}=0, \quad[\mathcal{F}, \mathcal{F}] \subset \mathcal{F} .
$$

Locally in $V$ the prescription of $\mathcal{F}$ is equivalent to prescribing a basis for $\mathcal{F}(V)$ of the form $\left\{L_{1}, \ldots, L_{n}, M_{1}, \ldots, M_{m}\right\}$, where the $L_{i}$ are of degree 0 , the $M_{i}$ are of degree 1, and which satisfy conditions (1.4) and (1.5) with coefficients that are sections of $\mathcal{B}_{\mathbb{C}}$.

Let $(X, \mathcal{A}, \alpha)$ be a real $C^{\infty}$ supermanifold of dimension $(N, M)$, where $N=2 n+l$ and $M=2 m+k$, with $l, k \geq 0$. Let $U$ be an open set in $X$ where supercoordinates $\left(t^{1}, \ldots, t^{2 n}, r^{1}, \ldots, r^{l}, \tau^{1}, \ldots, \tau^{2 m}, \varrho^{1}, \ldots, \varrho^{k}\right)$ are given and suppose we have complex derivations $\left\{L_{1}, \ldots, L_{n}, M_{1}, \ldots, M_{m}\right\}$ of $\mathcal{A}_{\mathbb{C}}$ which on $U$ can be written as

$$
\begin{aligned}
& L_{i}=\sum_{j=1}^{2 n} a_{i}^{j}(t, r, \tau, \varrho) \frac{\partial}{\partial t^{j}}+\sum_{j=1}^{2 m} b_{i}^{j}(t, r, \tau, \varrho) \frac{\partial}{\partial \tau^{j}}, \\
& M_{i}=\sum_{j=1}^{2 n} c_{i}^{j}(t, r, \tau, \varrho) \frac{\partial}{\partial t^{j}}+\sum_{j=1}^{2 m} d_{i}^{j}(t, r, \tau, \varrho) \frac{\partial}{\partial \tau^{j}},
\end{aligned}
$$

where the coefficients are sections of $\mathcal{A}_{\mathbb{C}}$. We say that this family forms a super integrable almost complex structure with parameters $(r, \varrho)$ on $(U, \mathcal{A}(U), \alpha)$ if the span of this collection is closed under supercommutators and the family $\left\{L_{1}, \ldots, L_{n}, \bar{L}_{1}, \ldots, \bar{L}_{n}, M_{1}, \ldots, M_{m}, \bar{M}_{1}, \ldots, \bar{M}_{m}\right\}$ is linearly independent. Note that such a collection defines a super integrable almost complex structure on the super submanifold $\left(U_{r}, \mathcal{A}_{r, \varrho}, \widehat{\alpha}\right)$, where $U_{r}$ is the submanifold of $U$ defined by freezing the value of the function $\widetilde{r}$, $\mathcal{A}_{r, \varrho}$ is the $\mathbb{Z}_{2}$ graded algebra generated by 1 and $\tau=\left(\tau^{1}, \ldots, \tau^{2 m}\right)$ over $C^{\infty}\left(U_{r}\right) \otimes \bigwedge^{*} \mathbb{R}_{\varrho}^{k}$, and $\widehat{\alpha}$ is the corresponding induced augmentation map.

ThEOREM 4. Let $\left\{L_{1}, \ldots, L_{n}, M_{1}, \ldots, M_{m}\right\}$ be a super integrable almost complex structure with parameters $(r, \varrho)$ on $(U, \mathcal{A}(U), \alpha)$. Then, on a possibly smaller open set $U$, there exists a $C^{\infty}$ change of the real supercoordinates of the form $\{x=x(t, r, \tau, \varrho), y=y(t, r, \tau, \varrho), r, \xi=\xi(t, r, \tau, \varrho), \eta=$ $\eta(t, r, \tau, \varrho), \varrho\}$ such that $(x, y, \xi, \eta)$ are real supercoordinates on $\left(U_{r}, \mathcal{A}_{r, \varrho}, \widehat{\alpha}\right)$. If we set $z^{j}=x^{j}+\sqrt{-1} y^{j}, \zeta^{j}=\xi^{j}+\sqrt{-1} \eta^{j}$, the derivations $\partial / \partial z^{j}, \partial / \partial \zeta^{k}$ form a basis for the original structure spanned by the $L_{j}, M_{k}$.

The nonparametric version of this result was proven in [8]. The authors 
[3] have given a proof of the nonparametric version of the above result in the much more complicated case in which the work must be done on one side of a weakly pseudoconvex boundary near a boundary point $p$. But when one is working in a full neighborhood of an interior point $p$, as we are now doing in this paper, all of the technical up-to-the-boundary difficulties encountered in [3] disappear. Hence the method of proof used there implies Theorem 4 immediately, since every step in the proof depends smoothly on parameters; e.g., we need only use the Dolbeault lemma for, say, a polydisc in $\mathbb{C}^{n}$, which can clearly be made to depend smoothly on parameters. Thus Theorem 4 is implicitly contained in [3], although it was not explicitly stated there.

3. Proof of Theorem 1. Consider a local basis $\left\{P_{1}, \ldots, P_{n+m}\right\}=$ $\left\{L_{1}, \ldots, L_{n}, M_{1}, \ldots, M_{m}\right\}$ for the sections of $\mathcal{H}$ with properties (1.4)-(1.6). The coefficients in (1.5) and (1.6) are elements of $\mathcal{A}_{\mathbb{C}}(U)$. Using only addition, subtraction, and complex conjugation, we see that the supercommutators $\left[\operatorname{Re} P_{i}, \operatorname{Re} P_{j}\right],\left[\operatorname{Re} P_{i}, \operatorname{Im} P_{j}\right]$ and $\left[\operatorname{Im} P_{i}, \operatorname{Im} P_{j}\right]$ are all linear combinations of the $\operatorname{Re} P_{i}, \operatorname{Im} P_{j}$ with real coefficients in $\mathcal{A}(U)$. Thus the subsheaf $\mathcal{J}$ spanned by these derivations is a super integrable distribution on $(U, \mathcal{A}(U), \alpha)$ of type $(2 n, 2 m)$. By Theorem 3 , given a point $p \in U$, there exists a neighborhood $V$ and supercoordinates $\left(t^{1}, \ldots, t^{2 n}, r^{1}, \ldots, r^{l}, \tau^{1}, \ldots\right.$, $\left.\tau^{2 m}, \varrho^{1}, \ldots, \varrho^{k}\right)$ such that $\mathcal{J}$ is spanned by the derivations $\partial / \partial t^{i}, \partial / \partial \tau^{k}$, $1 \leq i \leq 2 n, 1 \leq k \leq 2 m$.

From the definition of $\mathcal{J}$ it then follows that there are $C^{\infty}$ complex superfunction coefficients for which

$$
\begin{aligned}
L_{i} & =\sum_{j=1}^{2 n} a_{i}^{j}(t, r, \tau, \varrho) \frac{\partial}{\partial t^{j}}+\sum_{j=1}^{2 m} b_{i}^{j}(t, r, \tau, \varrho) \frac{\partial}{\partial \tau^{j}}, \\
M_{i} & =\sum_{j=1}^{2 n} c_{i}^{j}(t, r, \tau, \varrho) \frac{\partial}{\partial t^{j}}+\sum_{j=1}^{2 m} d_{i}^{j}(t, r, \tau, \varrho) \frac{\partial}{\partial \tau^{j}} .
\end{aligned}
$$

This means that on $(V, \mathcal{A}(V), \alpha)$ we have a super integrable almost complex structure with parameters $(r, \varrho)$. We now apply Theorem 4 to obtain real supercoordinates $(x, y, r, \xi, \eta, \varrho)$ such that the $\partial / \partial z^{j}, \partial / \partial \zeta^{k}$ span the same subsheaf as the one spanned by the $L_{j}, M_{k}$. This completes the proof of Theorem 1.

4. Proof of Theorem 2. Recall that locally there is a basis for the super complex Frobenius structure $\mathcal{D}$ of the form $\left\{L_{1}, \ldots, L_{n}, X_{1}, \ldots, X_{e}\right.$, $\left.M_{1}, \ldots, M_{m}, Y_{1}, \ldots, Y_{d}\right\}$ where the $L_{i}, X_{j}$ are even, the $M_{i}, Y_{j}$ are odd, and the $X_{i}, Y_{j}$ are real. From the integrability conditions $[\mathcal{D}, \mathcal{D}] \subset \mathcal{D},[\mathcal{D}, \overline{\mathcal{D}}] \subset$ $\mathcal{D}+\overline{\mathcal{D}}$ and their complex conjugates, it readily follows that 
$[\mathcal{D}+\overline{\mathcal{D}}, \mathcal{D}+\overline{\mathcal{D}}] \subset \mathcal{D}+\overline{\mathcal{D}}$ and $[\mathcal{D}+\overline{\mathcal{D}}, \overline{\mathcal{D}+\overline{\mathcal{D}}}] \subset \mathcal{D}+\overline{\mathcal{D}}$. This means that the real derivations $\left\{\operatorname{Re} L_{i}, \operatorname{Im} L_{i}, X_{j}, \operatorname{Re} M_{i}, \operatorname{Im} M_{i}, Y_{j}\right\}$ span an integrable super real distribution $\mathcal{J}$ on $(X, \mathcal{A}, \alpha)$ of type $(2 n+e, 2 m+d)$. Hence by Theorem 3 there is a neighborhood $U$ and a real supercoordinate system in $U$ with even supercoordinates $\left(q^{1}, \ldots, q^{2 n+e}, r^{1}, \ldots, r^{l}\right)$ and odd supercoordinates $\left(\varphi^{1}, \ldots, \varphi^{2 m+d}, \varrho^{1}, \ldots, \varrho^{k}\right)$ such that the $\partial / \partial q^{i}, \partial / \partial \varphi^{j} \operatorname{span} \mathcal{J}$. In term of these coordinates we can write

$$
\begin{array}{rlrl}
L_{i} & =A_{i}^{0 j} \frac{\partial}{\partial q^{j}}+B_{i}^{0 j} \frac{\partial}{\partial \varphi^{j}}, & X_{k} & =C_{k}^{0 j} \frac{\partial}{\partial q^{j}}+D_{k}^{0 j} \frac{\partial}{\partial \varphi^{j}}, \\
M_{i}=A_{i}^{1 j} \frac{\partial}{\partial q^{j}}+B_{i}^{1 j} \frac{\partial}{\partial \varphi^{j}}, & Y_{k}=C_{k}^{1 j} \frac{\partial}{\partial q^{j}}+D_{k}^{1 j} \frac{\partial}{\partial \varphi^{j}},
\end{array}
$$

with superfunction coefficients depending on $(q, r, \varphi, \varrho)$ and with the $C_{k}^{i j}$, $D_{k}^{i j}$ real. In effect we have solved for a local "big leaf" corresponding to constant "values" of the parameters $(r, \varrho)=\left(r^{1}, \ldots, r^{l}, \varrho^{1}, \ldots, \varrho^{k}\right)$.

For each value of these parameters we have the super distribution $\mathcal{D} \cap$ $\overline{\mathcal{D}} \subset \mathcal{D}+\overline{\mathcal{D}}$ spanned on $U$ by $\left\{X_{1}, \ldots, X_{e}, Y_{1}, \ldots, Y_{d}\right\}$. It is integrable of type $(e, d)$ because our given integrability condition implies that $[\mathcal{D} \cap$ $\overline{\mathcal{D}}, \mathcal{D} \cap \overline{\mathcal{D}}] \subset \mathcal{D} \cap \overline{\mathcal{D}}$ and $[\mathcal{D} \cap \overline{\mathcal{D}}, \overline{\mathcal{D} \cap \overline{\mathcal{D}}}] \subset \mathcal{D} \cap \overline{\mathcal{D}}$. Thus we have a real super distribution with parameters $(r, \varrho)$, so by Remark 1 we can find new real even supercoordinates $\left(p^{1}, \ldots, p^{2 n}, t^{1}, \ldots, t^{e}\right)$ to replace the $q$ 's, and new odd supercoordinates $\left(\pi^{1}, \ldots, \pi^{2 m}, \tau^{1}, \ldots, \tau^{d}\right)$ to replace the $\varphi$ 's, so that we can take as a new basis for $\mathcal{D} \cap \overline{\mathcal{D}}$ the supercommuting coordinate derivations

$$
X_{k}^{\prime}=\partial / \partial t^{k}, \quad k=1, \ldots, e, \quad Y_{l}^{\prime}=\partial / \partial \tau^{l}, \quad l=1, \ldots, d .
$$

In this new supercoordinate system $(p, t, r, \pi, \tau, \varrho)$ the $L_{i}, M_{j}$ are expressed in the form

$$
\begin{aligned}
L_{i} & =a_{i}^{0 j} \frac{\partial}{\partial p^{j}}+c_{i}^{0 j} \frac{\partial}{\partial t^{j}}+b_{i}^{0 j} \frac{\partial}{\partial \pi^{j}}+d_{i}^{0 j} \frac{\partial}{\partial \tau^{j}}, \\
M_{i} & =a_{i}^{1 j} \frac{\partial}{\partial p^{j}}+c_{i}^{1 j} \frac{\partial}{\partial t^{j}}+b_{i}^{1 j} \frac{\partial}{\partial \pi^{j}}+d_{i}^{1 j} \frac{\partial}{\partial \tau^{j}},
\end{aligned}
$$

with superfunction coefficients depending on $(p, t, r, \pi, \tau, \varrho)$. We can replace the $L_{i}, M_{j}$ by

$$
L_{i}^{\prime}=L_{i}-c_{i}^{0 k} X_{k}^{\prime}-d_{i}^{0 k} Y_{k}^{\prime}, \quad M_{i}^{\prime}=M_{i}-c_{j}^{1 k} X_{k}^{\prime}-d_{j}^{1 k} Y_{k}^{\prime},
$$

and obtain a new basis $\left\{L_{1}^{\prime}, \ldots, L_{n}^{\prime}, X_{1}^{\prime}, \ldots, X_{e}^{\prime}, M_{1}^{\prime}, \ldots, M_{m}^{\prime}, Y_{1}^{\prime}, \ldots, Y_{d}^{\prime}\right\}$ for $\mathcal{D}$ as well as a new basis $\left\{L_{1}^{\prime}, \ldots, L_{n}^{\prime}, \bar{L}_{1}^{\prime}, \ldots, \bar{L}_{n}^{\prime}, X_{1}^{\prime}, \ldots, X_{e}^{\prime}, M_{1}^{\prime}, \ldots, M_{m}^{\prime}\right.$, $\left.\bar{M}_{1}^{\prime}, \ldots, \bar{M}_{m}^{\prime}, Y_{1}^{\prime}, \ldots, Y_{d}^{\prime}\right\}$ for $\mathcal{D} \cap \overline{\mathcal{D}}$. It is now convenient to drop the primes 
and write

$$
\begin{aligned}
L_{i} & =a_{i}^{0 j} \frac{\partial}{\partial p^{j}}+b_{i}^{0 j} \frac{\partial}{\partial \pi^{j}}, & X_{k} & =\frac{\partial}{\partial t^{k}}, \\
M_{i} & =a_{i}^{1 j} \frac{\partial}{\partial p^{j}}+b_{i}^{1 j} \frac{\partial}{\partial \pi^{j}}, & Y_{l} & =\frac{\partial}{\partial \tau^{l}} .
\end{aligned}
$$

The advantage of this new basis is that, while the $X_{k}, Y_{l}$ supercommute among themselves, the supercommutators of the $L_{i}$ and $M_{j}$ are linear combinations of the $\partial / \partial p^{i}$ and $\partial / \partial \pi^{j}$ solely. From the integrability conditions, we conclude that $\left[L_{i}, L_{j}\right],\left[M_{i}, M_{j}\right]$ and $\left[L_{i}, M_{j}\right]$ are linear combinations of the $L_{k}$ and $M_{k}$ only; whereas the $\left[L_{i}, \bar{L}_{j}\right],\left[M_{i}, \bar{M}_{j}\right]$ and $\left[L_{i}, \bar{M}_{j}\right]$ are linear combinations of the $L_{k}, M_{k}$ and $\bar{L}_{k}, \bar{M}_{k}$ only, with $(t, r, \tau, \varrho)$ playing the role of parameters. Thus we have a local integrable super almost complex structure of dimension $(n, m)$ on each "small leaf" corresponding to constant "values" of the parameters $\left(t^{1}, \ldots, t^{e}, r^{1}, \ldots, r^{l}, \tau^{1}, \ldots, \tau^{d}, \varrho^{1}, \ldots, \varrho^{k}\right)$. Therefore we can apply Theorem 4 to obtain real new even supercoordinates $\left(x^{1}, \ldots, x^{n}, y^{1}, \ldots, y^{n}\right)$ to replace the $p$ 's, and new odd supercoordinates $\left(\xi^{1}, \ldots, \xi^{m}, \eta^{1}, \ldots, \eta^{m}\right)$ to replace the $\pi$ 's, so that the new superderivations

$$
L_{i}=\partial / \partial z^{i}, \quad i=1, \ldots, n, \quad M_{j}=\partial / \partial \zeta^{j}, \quad j=1, \ldots, m,
$$

span the super complex structure on each subleaf. Here the $X_{k}, Y_{l}$ are not changed, but they have new expressions in the new supercoordinates $(x, y, t, r, \xi, \eta, \tau, \varrho)$ of the form

$$
\begin{aligned}
X_{k} & =\frac{\partial}{\partial t^{k}}+\alpha_{k}^{0 j} L_{j}+\bar{\alpha}_{k}^{0 j} \bar{L}_{j}+\beta_{k}^{0 j} M_{j}+\bar{\beta}_{k}^{0 j} \bar{M}_{j}, \\
Y_{k} & =\frac{\partial}{\partial \tau^{k}}+\alpha_{k}^{1 j} L_{j}+\bar{\alpha}_{k}^{1 j} \bar{L}_{j}+\beta_{k}^{1 j} M_{j}+\bar{\beta}_{k}^{1 j} \bar{M}_{j},
\end{aligned}
$$

for some complex superfunction coefficients $\alpha_{k}^{i j}, \beta_{k}^{i j}$, which may depend on all the coordinates $(x, y, t, r, \xi, \eta, \tau, \varrho)$.

Consider a mixed bracket, such as $\left[L_{i}, X_{k}\right]$. From $[\mathcal{D}, \mathcal{D}] \subset \mathcal{D}$ and the form of the $X_{k}, Y_{l}$, we see that it must be a linear combination of the $L_{i}$, $M_{j}$ only, from which it follows that $L_{i} \bar{\alpha}_{k}^{0 j}=0$ and $L_{i} \bar{\beta}_{k}^{0 j}=0$. A similar consideration of the bracket $\left[M_{i}, X_{k}\right]$ shows that $M_{i} \bar{\alpha}_{k}^{0 j}=0$ and $M_{i} \bar{\beta}_{k}^{0 j}=0$. Likewise with $X_{k}$ replaced by $Y_{k}$. It follows that all the coefficients $\alpha_{k}^{i j}, \beta_{k}^{i j}$ are superholomorphic with respect to $(z, \xi)$. Note also that the $X_{k}, Y_{l}$ still supercommute. In order to complete the proof of the theorem we need the following

Lemma 1. Consider $L_{i}, X_{k}, M_{j}, Y_{l}$ as in (4.14), (4.15) where the $X_{k}$, $Y_{l}$ supercommute, and the coefficients $\alpha_{k}^{i j}, \beta_{k}^{i j}$ are $C^{\infty}$ holomorphic 
superfunctions with respect to $(z, \zeta)$. Then there is a $C^{\infty}$ change of supercoordinates of the form

$$
\begin{array}{lll}
t=s, & z=z(s, w, r, \sigma, \omega, \varrho), & r=r, \\
\tau=\sigma, & \zeta=\zeta(s, w, r, \sigma, \omega, \varrho), & \varrho=\varrho,
\end{array}
$$

such that all the $z$ 's and $\zeta$ 's are superholomorphic with respect to the $w$ 's and $\omega$ 's, and satisfy

$$
\begin{array}{rlrl}
\frac{\partial z^{j}}{\partial s^{k}} & =\alpha_{k}^{0 j}(s, z, r, \sigma, \zeta, \varrho), & & \frac{\partial \zeta^{j}}{\partial s^{k}}=\beta_{k}^{0 j}(s, z, r, \sigma, \zeta, \varrho), \\
\frac{\partial z^{j}}{\partial \sigma^{k}}=\alpha_{k}^{1 j}(s, z, r, \sigma, \zeta, \varrho), & \frac{\partial \zeta^{j}}{\partial \sigma^{k}}=\beta_{k}^{1 j}(s, z, r, \sigma, \zeta, \varrho),
\end{array}
$$

with initial conditions

$$
z^{j}(0,0)=w^{j}, \quad \zeta^{j}(0,0)=\omega^{j} .
$$

Here $s=\left(s^{1}, \ldots, s^{e}\right), \sigma=\left(\sigma^{1}, \ldots, \sigma^{d}\right)$.

Assuming the Lemma for the moment, we proceed to finish the proof of Theorem 2: we calculate the $X_{k}, Y_{k}$ in the new supercoordinate system $(w, s, r, \omega, \sigma, \varrho)$ and find that

$$
\begin{gathered}
\frac{\partial}{\partial s^{k}}=\frac{\partial}{\partial t^{k}}+\alpha_{k}^{0 j} \frac{\partial}{\partial z^{j}}+\bar{\alpha}_{k}^{0 j} \frac{\partial}{\partial \bar{z}^{j}}+\beta_{k}^{0 j} \frac{\partial}{\partial \zeta^{j}}+\bar{\beta}_{k}^{0 j} \frac{\partial}{\partial \bar{\zeta}^{j}}=X_{k}, \\
\frac{\partial}{\partial \sigma^{k}}=\frac{\partial}{\partial \tau^{k}}+\alpha_{k}^{1 j} \frac{\partial}{\partial z^{j}}+\bar{\alpha}_{k}^{1 j} \frac{\partial}{\partial \bar{z}^{j}}+\beta_{k}^{1 j} \frac{\partial}{\partial \zeta^{j}}+\bar{\beta}_{k}^{1 j} \frac{\partial}{\partial \bar{\zeta}^{j}}=Y_{k} .
\end{gathered}
$$

We also have

$$
\frac{\partial}{\partial w^{i}}=\frac{\partial z^{j}}{\partial w^{i}} L_{j}+\frac{\partial \zeta^{j}}{\partial w^{i}} M_{j}, \quad \frac{\partial}{\partial \omega^{i}}=\frac{\partial z^{j}}{\partial \omega^{i}} L_{i}+\frac{\partial \zeta^{j}}{\partial \omega^{i}} M_{j}
$$

by the superholomorphicity, and the above linear combinations preserve linear independence. Hence we may take $\left\{\partial / \partial w^{1}, \ldots, \partial / \partial w^{n}, \partial / \partial s^{1}, \ldots\right.$, $\left.\partial / \partial s^{e}, \partial / \partial \omega^{1}, \ldots, \partial / \partial \omega^{m}, \partial / \partial \sigma^{1}, \ldots, \partial / \partial \sigma^{d}\right\}$ as a new local basis for $\mathcal{D}$, and the proof of Theorem 2 is complete.

Proof of Lemma 1 . Using

$$
\bar{L}_{s} \alpha_{k}^{i j}=0, \quad \bar{L}_{s} \beta_{k}^{i j}=0, \quad \bar{M}_{s} \alpha_{k}^{i j}=0, \quad \bar{M}_{s} \beta_{k}^{i j}=0,
$$

and the fact that the $X_{k}, Y_{l}$ are real with $X_{k}$ even and $Y_{l}$ odd, we see that each of the relations $\left[X_{k}, X_{l}\right]=0,\left[X_{k}, Y_{l}\right]=0,\left[Y_{k}, Y_{l}\right]=0$ yields the vanishing of two expressions. Thus we are led to calculate the following six 
integrability conditions:

$$
\begin{aligned}
& \partial_{t^{k}} \alpha_{l}^{0 j}+\alpha_{k}^{0 s} L_{s} \alpha_{l}^{0 j}+\beta_{k}^{0 s} M_{s} \alpha_{l}^{0 j}=\partial_{t^{l}} \alpha_{k}^{0 j}+\alpha_{l}^{0 s} L_{s} \alpha_{k}^{0 j}+\beta_{l}^{0 s} M_{s} \alpha_{k}^{0 j}, \\
& \partial_{t^{k}} \beta_{l}^{0 j}+\alpha_{k}^{0 s} L_{s} \beta_{l}^{0 j}+\beta_{k}^{0 s} M_{s} \beta_{l}^{0 j}=\partial_{t^{l}} \beta_{k}^{0 j}+\alpha_{l}^{0 s} L_{s} \alpha_{k}^{0 j}+\beta_{l}^{0 s} M_{s} \beta_{k}^{0 j}, \\
& \partial_{t^{k}} \alpha_{l}^{1 j}+\alpha_{k}^{0 s} L_{s} \alpha_{l}^{1 j}+\beta_{k}^{0 s} M_{s} \alpha_{l}^{1 j}=\partial_{\tau^{l}} \alpha_{k}^{0 j}+\alpha_{l}^{1 s} L_{s} \alpha_{k}^{0 j}+\beta_{l}^{1 s} M_{s} \alpha_{k}^{0 j}, \\
& \partial_{t^{k}} \beta_{l}^{1 j}+\alpha_{k}^{0 s} L_{s} \beta_{l}^{1 j}+\beta_{k}^{0 s} M_{s} \beta_{l}^{1 j}=\partial_{\tau^{l}} \beta_{k}^{0 j}+\alpha_{l}^{1 s} L_{s} \beta_{k}^{0 j}+\beta_{l}^{1 s} M_{s} \beta_{k}^{0 j}, \\
& \partial_{\tau^{k}} \alpha_{l}^{1 j}+\alpha_{k}^{1 s} L_{s} \alpha_{l}^{1 j}+\beta_{k}^{1 s} M_{s} \alpha_{l}^{1 j}=-\partial_{\tau^{l}} \alpha_{k}^{1 j}-\alpha_{l}^{1 s} L_{s} \alpha_{k}^{1 j}-\beta_{l}^{1 s} M_{s} \alpha_{k}^{1 j}, \\
& \partial_{\tau^{k}} \beta_{l}^{1 j}+\alpha_{l}^{1 s} L_{s} \beta_{k}^{1 j}+\beta_{l}^{1 s} M_{s} \beta_{k}^{1 j}=-\partial_{\tau^{l}} \beta_{k}^{1 j}-\alpha_{l}^{1 s} L_{s} \beta_{k}^{1 j}-\beta_{l}^{1 s} M_{s} \beta_{k}^{1 j} .
\end{aligned}
$$

We now may use the holomorphicity to add to each of the above expressions, in respective order, the zero terms

$$
\begin{aligned}
& \bar{\alpha}_{k}^{0 s} \bar{L}_{s} \alpha_{l}^{0 j}+\bar{\beta}_{k}^{0 s} \bar{M}_{s} \alpha_{l}^{0 j}=\bar{\alpha}_{l}^{0 s} \bar{L}_{s} \alpha_{k}^{0 j}+\bar{\beta}_{l}^{0 s} \bar{M}_{s} \alpha_{k}^{0 j}, \\
& \bar{\alpha}_{k}^{0 s} \bar{L}_{s} \beta_{l}^{0 j}+\bar{\beta}_{k}^{0 s} \bar{M}_{s} \beta_{l}^{0 j}=\bar{\alpha}_{l}^{0 s} \bar{L}_{s} \beta_{k}^{0 j}+\bar{\beta}_{l}^{0 s} \bar{M}_{s} \beta_{k}^{0 j}, \\
& \bar{\alpha}_{k}^{0 s} \bar{L}_{s} \alpha_{l}^{1 j}+\bar{\beta}_{k}^{0 s} \bar{M}_{s} \alpha_{l}^{1 j}=\bar{\alpha}_{l}^{1 s} \bar{L}_{s} \alpha_{k}^{0 j}+\bar{\beta}_{l}^{1 s} \bar{M}_{s} \alpha_{k}^{0 j}, \\
& \bar{\alpha}_{k}^{0 s} \bar{L}_{s} \beta_{l}^{1 j}+\bar{\beta}_{k}^{0 s} \bar{M}_{s} \beta_{l}^{1 j}=\bar{\alpha}_{l}^{1 s} \bar{L}_{s} \beta_{k}^{0 j}+\bar{\beta}_{l}^{1 s} \bar{M}_{s} \beta_{k}^{0 j}, \\
& \bar{\alpha}_{k}^{1 s} \bar{L}_{s} \alpha_{l}^{1 j}+\bar{\beta}_{k}^{1 s} \bar{M}_{s} \alpha_{l}^{1 j}=-\bar{\alpha}_{l}^{1 s} \bar{L}_{s} \alpha_{k}^{1 j}-\bar{\beta}_{l}^{1 s} \bar{M}_{s} \alpha_{k}^{1 j}, \\
& \bar{\alpha}_{k}^{1 s} \bar{L}_{s} \beta_{l}^{1 j}+\bar{\beta}_{k}^{1 s} \bar{M}_{s} \beta_{l}^{1 j}=-\bar{\alpha}_{l}^{1 s} \bar{L}_{s} \beta_{k}^{1 j}-\bar{\beta}_{l}^{1 s} \bar{M}_{s} \beta_{k}^{1 j} .
\end{aligned}
$$

Recall that $z^{j}=x^{j}+\sqrt{-1} y^{j}$ and $\zeta^{j}=\xi^{j}+\sqrt{-1} \eta^{j}$. Let

$$
\alpha_{k}^{i j}=a_{k}^{i j}+\sqrt{-1} b_{k}^{i j}, \quad \beta_{k}^{i j}=c_{k}^{i j}+\sqrt{-1} d_{k}^{i j}
$$

be written in terms of their real and imaginary parts. The system to solve assumes the real form

$$
\begin{array}{llll}
\frac{\partial x^{j}}{\partial s^{k}}=a_{k}^{0 j}, & \frac{\partial y^{j}}{\partial s^{k}}=b_{k}^{0 j}, & \frac{\partial \xi^{j}}{\partial s^{k}}=c_{k}^{0 j}, & \frac{\partial \eta^{j}}{\partial s^{k}}=d_{k}^{0 j}, \\
\frac{\partial x^{j}}{\partial \sigma^{k}}=a_{k}^{1 j}, & \frac{\partial y^{j}}{\partial \sigma^{k}}=b_{k}^{1 j}, & \frac{\partial \xi^{j}}{\partial \sigma^{k}}=c_{k}^{1 j}, & \frac{\partial \eta^{j}}{\partial \sigma^{k}}=d_{k}^{1 j},
\end{array}
$$

with right hand sides superfunctions of the real supercoordinates $(x, y, s, r$, $\xi, \eta, \sigma, \varrho)$, and with initial conditions

$$
\begin{array}{ll}
x^{j}(0,0)=\operatorname{Re} w^{j}, & y^{j}(0,0)=\operatorname{Im} w^{j}, \\
\xi^{j}(0,0)=\operatorname{Re} \omega^{j}, & \eta^{j}(0,0)=\operatorname{Im} \omega^{j} .
\end{array}
$$

If we take the real and imaginary parts of our six integrability conditions with the terms added, as indicated above, we get twelve real integrability conditions, which, by inspection, are seen to be exactly what is needed to solve (4.18), (4.19) according to Corollary 1 . This gives us the $C^{\infty}$ solutions $z=z(s, w, r, \sigma, \omega, \varrho)$ and $\zeta=\zeta(s, w, r, \sigma, \omega, \varrho)$. 
We claim that these are superholomorphic with respect to the $w$ 's and $\omega^{\prime}$ 's: consider for example the variable $w^{1}$ and set $u^{j}=\partial_{w_{w}} z^{j}, v^{j}=\partial_{w_{w}} \zeta^{j}$. By differentiating we find that the $u^{j}, v^{j}$ satisfy the homogeneous system

$$
\begin{array}{lll}
\frac{\partial u^{j}}{\partial s^{k}}=\frac{\partial \alpha_{k}^{0 j}}{\partial z^{r}} u^{r}+\frac{\partial \alpha_{k}^{0 j}}{\partial \zeta^{r}} v^{r}, & \frac{\partial v^{j}}{\partial s^{k}}=\frac{\partial \beta_{k}^{0 j}}{\partial z^{r}} u^{r}+\frac{\partial \beta_{k}^{0 j}}{\partial \zeta^{r}} v^{r}, \\
\frac{\partial u^{j}}{\partial \sigma^{k}}=\frac{\partial \alpha_{k}^{1}}{\partial z^{r}} u^{r}+\frac{\partial \alpha_{k}^{1 j}}{\partial \zeta^{r}} v^{r}, & \frac{\partial v^{j}}{\partial \sigma^{k}}=\frac{\partial \beta_{k}^{1 j}}{\partial z^{r}} u^{r}+\frac{\partial \beta_{k}^{1 j}}{\partial \zeta^{r}} v^{r},
\end{array}
$$

with the initial conditions

$$
u^{j}(0,0)=0, \quad v^{j}(0,0)=0 .
$$

Hence $u^{j} \equiv 0$ and $v^{j} \equiv 0$ by the uniqueness part of Corollary 1. A similar argument applies to the variable $\omega^{1}$. Proceeding in this way by induction we obtain the result. This completes the proof of the Lemma.

\section{References}

[1] M. Eastwood and C. LeBrun, Thickening and supersymmetric extensions of complex manifolds, Amer. J. Math. 108 (1986), 1177-1192.

[2] P. Freund, Introduction to Supersymmetry, Cambridge Monographs Math. Phys., Cambridge 1986.

[3] C. D. Hill and S. R. Simanca, Newlander-Nirenberg theorem on supermanifolds with boundary, preprint, 1990.

[4] B. Kostant, Graded manifolds, graded Lie algebras, and prequantization, in: Lecture Notes in Math. 570, Springer, 1977, 177-306.

[5] C. LeBrun and M. Rothstein, Moduli of super Riemann surfaces, Comm. Math. Phys. 117 (1988), 159-176.

[6] D. A. Leites, Introduction to the theory of supermanifolds, Russian Math. Surveys 35 (1980), 3-57.

[7] Yu. I. Manin, Gauge Field Theory and Complex Geometry, Grundlehren Math. Wiss. 289, Springer, 1988 (Russian original published by Nauka, Moscow 1984).

[8] A. McHugh, A Newlander-Nirenberg theorem for super-manifolds, J. Math. Phys. 30 (5) (1989), 1039-1042.

[9] L. Nirenberg, A Complex Frobenius Theorem, Seminars on Analytic Functions, Vol. 1, Institute of Advanced Study, Princeton 1958.

DEPARTMENT OF MATHEMATICS STATE UNIVERSITY OF NEW YORK STONY BROOK, NEW YORK 11794 U.S.A. 